\title{
ON THE NUMBER OF HAMILTONIAN CIRCUITS IN THE ${ }_{n}$-CUBE
}

\author{
E. DIXON AND S. GOODMAN
}

ABSTRACT. Improved upper and lower bounds are found for the number of hamiltonian circuits in the $n$-cube.

I. Introduction. The $n$-cube $Q_{n}$ is an undirected $n$-regular graph with $2^{n}$ nodes. Each node is labeled $a_{n} a_{n-1} \cdots a_{1}$, where each $a_{i}$ is either 0 or 1. Two nodes of $Q_{n}$ are adjacent if and only if their binary representations differ in exactly one place. A hamiltonian circuit is a closed alternating succession of adjacent nodes and edges in which each node of the graph is visited exactly once. Two hamiltonian circuits are said to be distinct if they are not identical. The number of distinct hamiltonian circuits in $Q_{n}$ will be denoted by $h(n)$. Figure 1 shows $Q_{2}$ and $Q_{3}$ along with their values of $h(n)$.

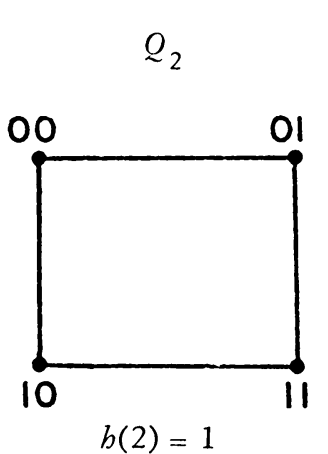

\section{$Q_{3}$}

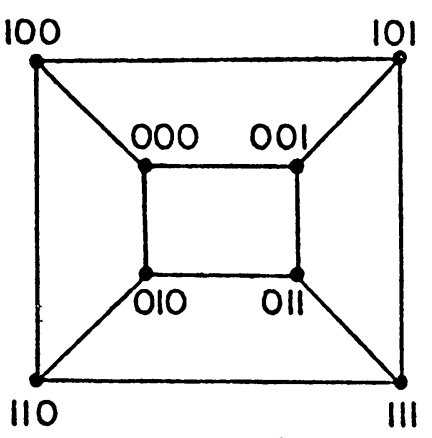

Figure 1. $Q_{2}$ and $Q_{3}$

A problem of considerable interest is that of determining the exact value of $h(n)$ for $n>3$. This problem arises in switching theory when trying to minimize errors in analog-digital systems (cf. [1], [2]). As yet only coarse bounds are known, and the purpose of this paper will be to derive better upper and lower bounds for $h(n)$.

Received by the editors March 21, 1974 and, in revised form, April 6, 1974. AMS (MOS) subject classifications (1970). Primary 05A20, 05C 35. node.

Key words and phrases. Hamiltonian circuit, $n$-cube, undirected graph, edge, 
IT. Lower bounds. In [3] Douglas derived the result that

$$
b(n)>C(\sqrt[7]{18})^{2^{n}}, \quad n \geq 2 .
$$

In deriving our lower bound we shall make use of the following lemma from Abbott [4].

Lemma 1. If $T_{n}$ denotes the number of distinct hamiltonian circuits which traverse a given edge of $Q_{n}$, then

$$
T_{n}=2 b(n) / n \text {. }
$$

The following lemma is a restatement of the definition of $Q_{n}$ and of an observation by Gilbert [2].

Lemma 2. Let $G_{1}$ and $G_{2}$ be two copies of $Q_{n-1}$. Then the graph of $Q_{n}$ can be constructed by connecting each node in $G_{1}$ to the node in $G_{2}$ that has the same labeling.

Since $Q_{n}$ has $2^{n}$ nodes, each with degree $n$, there are $n 2^{n} / 2=$ $n 2^{n-1}$ edges in $Q_{n}$. Now keeping the above construction of $Q_{n}$ in mind, consider the following class of hamiltonian circuits. In $G_{1}$ consider any hamiltonian circuit traversing some fixed edge (there are $T_{n}$ such circuits), and any hamiltonian circuit in $G_{2}$ traversing the corresponding edge there. If we now include the two edges connecting $G_{1}$ to $G_{2}$ through these four nodes and exclude the two original edges we will now have a hamiltonian circuit on $Q_{n}$. Since there are $(n-1) 2^{n-1 / 2}$ ways to choose the first edge we see that

$$
b(n)>(n-1) 2^{n-1} T^{2}(n-1) / 2 .
$$

This is similar to a result obtained by Abbott [4].

Now using the result of Lemma 1 we get

$$
b(n) \geq 2^{n} b^{2}(n-1) /(n-1) .
$$

Using this relation and a known value for $b(n)$ (for $n=3, b(3)=6$ ) we can solve this recurrence relation and get a lower bound on $h(n)$. Thus we see that

$$
b(4) \geq 2^{4}(6)^{2} / 3, \quad b(5) \geq\left(2^{5} / 4\right)\left[2^{4}(6)^{2} / 3\right]^{2}, \cdots
$$

which leads to the following form: 


$$
b(n) \geq \frac{2^{\left[\sum_{i=1}^{n-3}(n+1-i) 2^{i-1}\right]} 6^{2^{n-3}}}{\Pi_{i=1}^{n-3}(n-i)^{2^{i-1}}}, \quad n \geq 4 .
$$

Simplification of (4) leads to

$$
b(n) \geq \frac{2^{\left[2^{n-3}+2^{n-1}-n-2\right]} 6^{2^{n-3}}}{\Pi_{i=1}^{n-3}(n-i)^{2^{i-1}}}, \quad n \geq 4 .
$$

The construction used to derive (3) is called composition in [2]. We note that the lower bound (5) applies to a special kind of hamiltonian circuit called ultracomposite in [2]. The improved bound in (8) below does not apply to this class because some of the hamiltonian circuits in $Q_{4}$ are not ultracomposite.

Some manipulations are necessary before we can compare (5) with (1). Douglas' bound can be written as:

$$
b(n)=C(\sqrt[7]{18})^{2^{n}}=C a^{2^{n}}, \text { where } b(n) \text { is the actual bound, }
$$

so we now have

$$
b(n+1)=C a^{2^{n+1}}=C\left(a^{2^{n}}\right)^{2}=b^{2}(n) / C .
$$

Thus, we see that Douglas' bound satisfies the recurrence relation

$$
l(n)=b^{2}(n-1) / C
$$

where $C$ is a constant (about 1/5.2). Our recurrence relation is of the form

$$
b_{1}(n)=C(n) b_{1}^{2}(n-1)
$$

where $C(n)$ is an increasing function of $n$. For $n=4, C(4)>1 / C$ and $b_{1}(3)$ $=6>b(3)$ and therefore for all $n \geq 4$, (7) will dominate (6), hence (5) will be a better bound than (1).

If we use the results from $[5]$ on $Q_{4}$, we find that $h(4)=1,344$. This value can also be deduced from the symmetry properties of the hamiltonian circuits in $Q_{4}$ as developed by Gilbert in [2]. Now using this as a base we get

$$
b(n)>\frac{2^{\left[2^{n-2}+2^{n-3}-2-n\right]}(1,344)^{2^{n-4}}}{\prod_{i=0}^{n-5}(n-1-i)^{2^{i}}}, \quad n \geq 5,
$$

which is a significant improvement over (5). 
III. Upper bounds. It is stated in [1] that the best known upper bound for $h(n)$ is

$$
b(n)<n^{2^{n}}, \quad n \geq 4 .
$$

We now derive an improved upper bound using an argument first suggested to us by Gilbert [6].

Let each node in $Q_{n}$ be represented by the binary $n$-tuple $a_{n} a_{n-1} \cdots a_{1}$ where each $a_{i}$ is either 0 or 1 . A node will be called odd if the sum $a_{1}+$ $a_{2}+\cdots+a_{n}$ is odd, and even otherwise. It is easily verified that each edge in $Q_{n}$ joins an odd node to an even node. The edges in any particular hamiltonian circuit can be specified by giving the edge pairs incident at each even node. At each of the $2^{n-1}$ even nodes there are $\left(\begin{array}{c}n \\ 2\end{array}\right)$ possible edge pairs. For any hamiltonian circuit selection of edge pairs for all but one of the even nodes uniquely determines the two edges incident to the remaining even node. Therefore we have

$$
b(n) \leq[n(n-1) / 2]^{2^{n-1}-1} .
$$

IV. Concluding remarks. Some numerical calculations based on equations (1), (8), (9), and (10) are shown in Table 1. Although equations (8) and (10) represent a considerable tightening on the bounds for $h(n)$, there is still much room for improvement.

Table 1

\begin{tabular}{|c||l|l||l|l|}
\hline$n$ & $\begin{array}{l}\text { Old } \\
\text { lower (1) }\end{array}$ & $\begin{array}{l}\text { new } \\
\text { lower (8) }\end{array}$ & $\begin{array}{l}\text { old } \\
\text { upper (9) }\end{array}$ & $\begin{array}{l}\text { new } \\
\text { upper (10) }\end{array}$ \\
\hline 5 & $1.03 \times 10^{5}$ & $1.45 \times 10^{7}$ & $2.33 \times 10^{22}$ & $1.00 \times 10^{15}$ \\
\hline 6 & $5.50 \times 10^{10}$ & $2.67 \times 10^{15}$ & $6.33 \times 10^{49}$ & $2.88 \times 10^{36}$ \\
\hline 7 & $1.57 \times 10^{22}$ & $1.52 \times 10^{32}$ & $1.49 \times 10^{108}$ & $1.99 \times 10^{83}$ \\
\hline 8 & $1.28 \times 10^{45}$ & $8.50 \times 10^{65}$ & $1.55 \times 10^{231}$ & $6.24 \times 10^{184}$ \\
\hline 9 & $8.52 \times 10^{90}$ & $4.62 \times 10^{133}$ & $3.76 \times 10^{488}$ & $7.29 \times 10^{396}$ \\
\hline 10 & $3.77 \times 10^{182}$ & $2.43 \times 10^{269}$ & $1.0 \times 10^{1024}$ & $5.82 \times 10^{830}$ \\
\hline
\end{tabular}




\section{REFERENCES}

1. V. Klee, Long paths and circuits on polytopes, Convex Polytopes, Pure and Appl. Math., vol. 16, Interscience, New York, 1967, pp. 356-389. MR 37 \#2085.

2. E. N. Gilbert, Gray codes and paths on the n-cube, Bell System Tech. J. 37 (1958), 815-826. MR 20 \#792.

3. R. J. Douglas, $A$ note on a theorem of $H$. L. Abbott, Canad. Math. Bull. 13 (1970), 79-81. MR 43 \#6117.

4. H. L. Abbott, Hamiltonian circuits and paths on the n-cube, Canad. Math. Bull. 9 (1966), 557-562. MR 34 \#7395.

5. E. Dixon and S. Goodman, An algorithm for finding all the hamiltonian circuits and two factors in an arbitrary directed or undirected graph, DAMACS Tech. Rept., 3-73, University of Virginia, Charlottsville, Va., 1973.

6. E. N. Gilbert, Private communication.

DEPARTMENT OF APPLIED MATHEMATICS AND COMPUTER SCIENCE, UNIVERSITY OF VIRGINIA, CHARLOTTESVILLE, VIRGINIA 22901 (Current address of S. Goodman)

Current address (E. Dixon): TRW Systems Group, Redonda Beach California 90278

\section{ERRATUM}

The following abstract is to an article which appeared in the January issue of the Proceedings on pages 41-44 under the subject classification Algebra and Number Theory. It should have appeared in the Combinatorics section.

\section{COUNTING PATTERNS WITH A GIVEN AUTOMORPHISM GROUP}

\section{DENNIS E. WHITE}

ABSTRACT. A formula, anal ogous to the classical Burnside lemma, is developed which counts orbit representatives from a set under a group action with a given stabilizer subgroup conjugate class. This formula is applied in a manner analogous to a proof of Pólya's theorem to obtain an enumeration of patterns with a given automorphism group. 